\title{
CUPID-0: a cryogenic calorimeter with particle identification for double beta decay search
}

\author{
Fabio Bellini* \\ Sapienza Università di Roma and INFN Roma \\ E-mail: fabio.bellinieromal.infn.it
}

\begin{abstract}
With their excellent energy resolution, efficiency, and intrinsic radio-purity, cryogenic calorimeters are primed for the search for neutrino-less Double Beta Decay (0vDBD). The sensitivity of these devices could be further increased by discriminating the dominant $\alpha$ background from the expected $\beta$ like signal. The CUPID-0 experiment aims at demonstrating that the measurement of the scintillation light produced by the absorber crystals allows for particle identification and, thus, for a complete rejection of the $\alpha$ background. The CUPID-0 detector, assembled in 2016 and now in commissioning phase, consists of $26 \mathrm{Zn}^{82} \mathrm{Se}$ scintillating calorimeters for about $2 \cdot 10$ $250 v$ DBD emitters. Here we present the preliminary results obtained with the detector and the perspectives for a next generation project.
\end{abstract}

The European Physical Society Conference on High Energy Physics

5-12 July, 2017

Venice

${ }^{*}$ Speaker. 
Neutrino-less Double Beta Decay (0vDBD) is a hypothesized nuclear transition in which a nucleus decays emitting only two electrons. This process cannot be accommodated in the Standard Model, as the absence of emitted neutrinos would violate the lepton number conservation. For this reason, its observation would have several implications for particle physics, astrophysics and cosmology. For the $0 v \mathrm{DBD}$ to occur neutrinos must be Majorana particles. This means that, in contrast to all the other known fermions, they must coincide with their own antiparticles.

Among the several experimental approaches proposed for the search for $0 \mathrm{vDBD}$, cryogenic calorimeters (historically called bolometers [1]) stand out for the possibility of achieving excellent energy resolution $(\sim 0.1 \%)$, efficiency $(\geq 80 \%)$ and intrinsic radio-purity. Moreover, the crystals operated as bolometers, can be grown starting from most of the $0 \mathrm{vDBD}$ emitters, enabling the test of different nuclei.

The state of the art of the bolometric technique is represented by CUORE (Cryogenic Underground Observatory for Rare Events [2]), an experiment composed by 988 bolometers for a total mass of $741 \mathrm{~kg}$, presently in data taking at Laboratori Nazionali del Gran Sasso (LNGS, Italy). Preliminary results show that the target of the experiment in terms of energy resolution $(5 \mathrm{keV}$ at $2.6 \mathrm{MeV})$ and background $\left(10^{-2}\right.$ counts $/ \mathrm{keV} / \mathrm{kg} / \mathrm{y}$ in the ROI) are within the scope thus implying CUORE will reach a $90 \%$ C.L. exclusion sensitivity of $9 \times 10^{25} \mathrm{yr}$ in 5 years of live-time [3].

Next-generation experiments plan to increase this sensitivity by at least one order of magnitude. The main challenges will consist in a further increase of the mass (achievable via isotopic enrichment of the source) as well as a further lowering of the background level in the region of interest that, according to the experience of the CUORE collaboration, is dominated by $\alpha$ particles [4]. These are the goals of the CUPID (Cuore Upgrade with Particle IDentification [5, 6]) interest group, that is envisioning a ton-scale experiment made of isotopically enriched crystals with a background close to zero in the region of interest.

Several groups are working on different technologies to prove the feasibility of CUPID. Some of them are trying to develop an active background rejection tool for the $\mathrm{TeO}_{2}$ crystals (see Refs [7, $8,9,10,11,12,13,14]$ and references therein).

The technology presented in this contribution is based on scintillating bolometers. The different properties of the light emission for electrons and $\alpha$ particles enable event-by-event rejection of $\alpha$ interactions, suppressing the overall background in the region of interest for NDBD of about one order of magnitude. Different compounds were tested: $\mathrm{ZnSe}[15,16], \mathrm{ZnMoO}_{4}$ [17, 18, 19 , 20, 21, 22, 23], $\mathrm{Li}_{2} \mathrm{MoO}_{4}[24,25,26]$. The main difficulty for the $\mathrm{R} \& \mathrm{D}$ activities exploiting new compounds is the development of a crystal with low intrinsic contaminations, high-quality and high production yield, which is crucial when dealing with the expensive enriched material.

Among the several options, the CUPID-0 collaboration chose enriched ZnSe crystals for the study of ${ }^{82} \mathrm{Se} 0 v \mathrm{DBD}$. The goal of this medium-scale experiment is proving that the simultaneous read-out of light and heat allows to reject the dominant source of background for calorimetric detectors, setting a first important milestone in view of CUPID.

\section{CUPID-0}

The CUPID-0 detector is an array of ZnSe crystals arranged in 5 columns using a (mainly) copper structure, that holds the detectors in position and provides the thermal link to a dilution 
refrigerator operating at about $10 \mathrm{mK}$. The array comprises 24 crystals $95.4 \%$ enriched in ${ }^{82} \mathrm{Se}$ (total mass of $9.65 \mathrm{~kg}$, corresponding to $5.28 \mathrm{~kg}$ of ${ }^{82} \mathrm{Se}$ ) and two natural crystals (total mass of $0.85 \mathrm{~kg}$, corresponding to $40 \mathrm{~g}$ of ${ }^{82} \mathrm{Se}$ ). Details about the production of enriched $\mathrm{Zn}^{82} \mathrm{Se}$ crystals can be found in Ref [27]. The light detector is a $170 \mu \mathrm{m}$-thick Ge disk coated on one face with a 60 $\mathrm{nm}$ layer of $\mathrm{SiO}_{2}$ to enhance the light collection and operated as a bolometer itself. Each device is instrumented with a NTD-Ge thermistor sensor and the crystal is read by one light detector at the top and one at the bottom.

The CUPID-0 detector was commissioned in early 2017 and it is now in data taking. All the detectors are working correctly. In the first data-taking period we obtained an average FWHM energy resolution of $25 \mathrm{keV}$ at $2998 \mathrm{keV}$. Results concerning the background rejection capability will be released in Fall 2017.

\section{Acknowledgements}

This work was partially supported by the LUCIFER experiment, funded by ERC under the European Union's Seventh Framework Programme (FP7/2007-2013)/ERC grant agreement n. 247115, funded within the ASPERA 2nd Common Call for R\&D Activities. We are particularly grateful to M. Iannone for its help in all the stages of the detector construction, to M. Guetti for the assistance in the cryogenic operations and to the mechanical workshop of LNGS (in particular E. Tatananni, A. Rotilio, A. Corsi, and B. Romualdi) for continuous and constructive help in the overall set-up design.

\section{References}

[1] E. Fiorini and T.O. Niinikoski, Nucl. Instr. and Meth. A 224, 83 (1984).

[2] D. R. Artusa et al. [CUORE Collaboration], Adv. High Energy Phys. 2015, 879871 (2015), http://dx.doi.org/10.1155/2015/879871

[3] C. Alduino et al. [CUORE Collaboration], Eur. Phys. J. C77 8, 532 (2017), http://dx.doi.org/10.1140/epjc/s10052-017-5098-9

[4] C. Alduino et al. [CUORE Collaboration], Eur.Phys.J. C77 8, 543 (2017), http://dx.doi.org/10.1140/epjc/s10052-017-5080-6

[5] G. Wang, et al. "R\&D towards CUPID (CUORE Upgrade with Particle IDentification) " arXiv:1504.03612. (2015).

[6] G. Wang, et al. "CUPID: CUORE (Cryogenic Underground Observatory for Rare Events) Upgrade with Particle IDentification" arXiv:1504.03599 (2015).

[7] N. Casali, et al. Eur. Phys. J. C 75 (1) 12 (2015), http://dx.doi.org/10.1140/epjc/s10052-014-3225-4

[8] K. Schaeffner, et al. Astropart. Phys. 69 30-36 (2015), http://dx.doi.org/10.1016/j.astropartphys.2015.03.008

[9] M. Willers, et al. JINST 10 (03) P03003 (2015), http://dx.doi.org/10.1088/1748-0221/10/03/P03003

[10] M. Biassoni, et al. Eur. Phys. J. C 75 (10) 480 (2015), http://dx.doi.org/10.1140/epjc/s10052-015-3712-2 
[11] E. Battistelli, et al. Eur. Phys. J. C 75 (8) (2015), http://dx.doi.org/10.1140/epjc/s10052-015-3575-6

[12] L. Cardani, et al. Appl. Phys. Lett. 107093508 (2015), http://dx.doi.org/10.1063/1.4929977

[13] F. Bellini et al. Appl. Phys. Lett. 110033504 (2017), http://dx.doi.org/10.1063/1.4974082

[14] D. R. Artusa et al. Phys. Lett. B 767321 (2017), http://dx.doi.org/10.1016/j.physletb.2017.02.011

[15] C. Arnaboldi, et al. Astropart. Phys. 34, 344 (2011), http://dx.doi.org/10.1016/j.astropartphys.2010.09.004

[16] J.W. Beeman, et al. JINST 8, P05021 (2013), http://dx.doi.org/10.1088/1748-0221/8/05/P05021

[17] L. Gironi, et al. JINST 5, P11007 (2010), http://dx.doi.org/10.1088/1748-0221/5/11/P11007

[18] J.W. Beeman, et al. Eur. Phys. J. C72, 2142 (2012), http://dx.doi.org/10.1140/epjc/s10052-012-2142-7

[19] J.W. Beeman, et al. Astropart. Phys. 35, 813 (2012), http://dx.doi.org/10.1016/j.astropartphys.2012.02.013

[20] J.W. Beeman, et al. Phys. Lett. B 710, 318 (2012), http://dx.doi.org/10.1016/j.physletb.2012.03.009

[21] L. Cardani, et al. J. Phys. G41, 075204 (2014), http://dx.doi.org/10.1088/0954-3899/41/7/075204

[22] E. Armengaud, et al. JINST 10(05), P05007 (2015), http://dx.doi.org/10.1088/1748-0221/10/05/P05007

[23] L. Berge, et al. JINST 9, P06004 (2014), http://dx.doi.org/10.1088/1748-0221/9/06/P06004

[24] L. Cardani, et al. JINST 8, P10002 (2013), http://dx.doi.org/10.1088/1748-0221/8/10/P10002

[25] T.B. Bekker, et al. Astropart. Phys. 72, 38 (2016), http://dx.doi.org/10.1016/j.astropartphys.2015.06.002

[26] E. Armengaud et al., arXiv:1704.01758.

[27] I. Dafinei et al., Journ. of Crystal Growth 475 158-170 (2017), http://dx.doi.org/10.1016/j.jcrysgro.2017.06.013

[28] D. R. Artusa et al., Eur. Phys. J. C 76 no.7, 364 (2016), http://dx.doi.org/10.1140/epjc/s10052-016-4223-5 